\title{
Computation of Positive Solutions for Nonlinear Impulsive Integral Boundary Value Problems with $p$-Laplacian on Infinite Intervals
}

\author{
Xingqiu Zhang \\ School of Mathematics, Liaocheng University, Liaocheng, Shandong 252059, China \\ Correspondence should be addressed to Xingqiu Zhang; zhxq197508@163.com
}

Received 14 October 2012; Accepted 11 January 2013

Academic Editor: Patricia J. Y. Wong

Copyright (C) 2013 Xingqiu Zhang. This is an open access article distributed under the Creative Commons Attribution License, which permits unrestricted use, distribution, and reproduction in any medium, provided the original work is properly cited.

This paper deals with the existence and iteration of positive solutions for nonlinear second-order impulsive integral boundary value problems with $p$-Laplacian on infinite intervals. Our approach is based on the monotone iterative technique.

\section{Introduction}

The theory of impulsive differential equations has been emerging as an important area of investigation in recent years. It has been extensively applied to biology, biologic medicine, optimum control in economics, chemical technology, population dynamics, and so on. It is much richer because all the structure of its emergence has deep physical background and realistic mathematical model and coincides with many phenomena in nature. For an introduction of the basic theory of impulsive differential equations in $R^{n}$, the reader is referred to see Lakshmikantham et al. $[1,2]$, Samoilenko and Perestyuk [3], and the references therein.

Boundary value problems on infinite intervals arise quite naturally in the study of radially symmetric solutions of nonlinear elliptic equations and models of gas pressure in a semi-infinite porous medium; see [4-7], for example. In a recent paper [8], by means of a fixed-point theorem due to Avery and Peterson, Li and Nieto obtained some new results on the existence of multiple positive solutions for the following multipoint boundary value problem with a finite number of impulsive times on an infinite interval:

$$
\begin{gathered}
u^{\prime \prime}(t)+q(t) f(t, u(t))=0, \\
\forall 0<t<\infty, \quad t \neq t_{k}, \quad k=1,2, \ldots, p,
\end{gathered}
$$

$$
\begin{aligned}
\Delta u\left(t_{k}\right) & =I_{k}\left(u\left(t_{k}\right)\right), \quad k=1,2, \ldots, p, \\
u(0) & =\sum_{i=1}^{m-2} \alpha_{i} u\left(\xi_{i}\right), \quad u^{\prime}(\infty)=0,
\end{aligned}
$$

where $f \in C([0,+\infty) \times[0,+\infty),[0,+\infty)), I_{k} \in C([0$, $+\infty),[0,+\infty)), u^{\prime}(\infty)=\lim _{t \rightarrow \infty} u^{\prime}(t), 0<\xi_{1}<\xi_{2}<\cdots<$ $\xi_{m-2}<+\infty, 0<t_{1}<t_{2}<\cdots<t_{p}<+\infty$, and $q \in C([0,+\infty),[0,+\infty))$.

Boundary value problems with integral boundary conditions for ordinary differential equations arise in different fields of applied mathematics and physics such as heat conduction, chemical engineering, underground water flow, thermoelasticity, and plasma physics. Moreover, boundary value problems with Riemann-Stieltjes integral conditions constitute a very interesting and important class of problems. They include two-point, three-point, and multipoint boundary value problems as special cases; see [9-14]. For boundary value problems with other integral boundary conditions and comments on their importance, we refer the reader to the papers [11-20] and the references therein.

There are relatively few papers available for integral boundary value problems for impulsive differential equations on an infinite interval with an infinite number of impulsive times up to now. In [21], Zhang et al. investigated the existence 
of minimal nonnegative solution for the following secondorder impulsive differential equation

$$
\begin{gathered}
-x^{\prime \prime}(t)=f\left(t, x(t), x^{\prime}(t)\right)=0, \quad t \in J, t \neq t_{k}, \\
\left.\Delta x\right|_{t=t_{k}}=I_{k}\left(x\left(t_{k}\right)\right), \quad k=1,2, \ldots, \\
\left.\Delta x^{\prime}\right|_{t=t_{k}}=\bar{I}_{k}\left(x\left(t_{k}\right)\right), \quad k=1,2, \ldots, \\
x(0)=\int_{0}^{+\infty} g(t) x(t) \mathrm{d} t, \quad x^{\prime}(\infty)=0,
\end{gathered}
$$

where $f \in C(J \times J \times J, J), I_{k} \in C(J, J), \bar{I}_{k} \in C(J, J), J=[0,+\infty)$, $0<t_{1}<t_{2}<\cdots<t_{k}<\cdots, t_{k} \rightarrow \infty$, and $g(t) \in L[J, J]$ with $\int_{0}^{+\infty} g(t) \mathrm{d} t<1 .\left.\Delta x\right|_{t=t_{k}}$ denotes the jump of $x(t)$ at $t=t_{k}$, that is,

$$
\left.\Delta x\right|_{t=t_{k}}=x\left(t_{k}^{+}\right)-x\left(t_{k}^{-}\right)
$$

where $x\left(t_{k}^{+}\right)$and $x\left(t_{k}^{-}\right)$represent the right-hand limit and lefthand limit of $x(t)$ at $t=t_{k}$, respectively. $\left.\Delta x^{\prime}\right|_{t=t_{k}}$ has a similar meaning to $x^{\prime}(t)$.

In the past few years, the existence and the multiplicity of bounded or unbounded positive solutions to nonlinear differential equations on infinite intervals have been studied by several different techniques; we refer the reader to [5$8,21-29]$ and the references therein. However, most of these papers only considered the existence of positive solutions under various boundary value conditions. Seeing such a fact, a natural question which arises is "how can we find the solutions when they are known to exist?" More recently, Ma et al. [30] and Sun et al. [31, 32] established iterative schemes for approximating the solutions for some boundary value problems defined on finite intervals by virtue of the iterative technique.

However, to the author's knowledge, the corresponding theory for impulsive integral boundary value problems with $p$-Laplacian operator and infinite impulsive times on infinite intervals has not been considered till now. Motivated by previous papers, the purpose of this paper is to obtain the existence of positive solutions and establish a corresponding iterative scheme for the following impulsive integral boundary value problem of second-order differential equation with $p$-Laplacian on an infinite interval

$$
\begin{gathered}
\left(\varphi_{p}\left(x^{\prime}(t)\right)\right)^{\prime}+q(t) f\left(t, x(t), x^{\prime}(t)\right)=0, \quad t \in J_{+}^{\prime}, \\
\left.\Delta x\right|_{t=t_{k}}=I_{k}\left(x\left(t_{k}\right)\right), \quad k=1,2, \ldots, \\
x(0)=\int_{0}^{+\infty} g(t) x(t) \mathrm{d} t, \quad x^{\prime}(\infty)=x_{\infty},
\end{gathered}
$$

where $\varphi_{p}(s)=|s|^{p-2} s, p>1, J=[0,+\infty), J_{+}=(0,+\infty), J_{+}^{\prime}=$ $J_{+} \backslash\left\{t_{1}, t_{2}, \ldots, t_{k}, \ldots\right\}, 0<t_{1}<t_{2}<\cdots<t_{k}<\cdots, t_{k} \rightarrow \infty$, and $g(t) \in L[J, J]$ with $\int_{0}^{+\infty} g(t) \mathrm{d} t<1, \int_{0}^{+\infty} \operatorname{tg}(t) \mathrm{d} t<+\infty$, and $0 \leq x^{\prime}(\infty)=\lim _{t \rightarrow+\infty} x^{\prime}(t)$.
It is clear that

$$
\begin{aligned}
& \varphi_{p}(s+t) \\
& \quad \leq \begin{cases}2^{p-1}\left(\varphi_{p}(s)+\varphi_{p}(t)\right), & p \geq 2, s, t>0, \\
\varphi_{p}(s)+\varphi_{p}(t), & 1<p<2, s, t>0,\end{cases} \\
& \varphi_{p}^{-1}(s+t) \\
& \quad \leq \begin{cases}2^{1 /(p-1)}\left(\varphi_{p}^{-1}(s)+\varphi_{p}^{-1}(t)\right), & p \geq 2, s, t>0, \\
\varphi_{p}^{-1}(s)+\varphi_{p}^{-1}(t), & 1<p<2, s, t>0 .\end{cases}
\end{aligned}
$$

Throughout this paper, we adopt the following assumptions.

$\left(\mathrm{H}_{1}\right) f(t, u, v) \in C(J \times J \times J, J), f(t, 0,0) \quad \equiv \quad 0$ on any subinterval of $J$, and when $u, v$ are bounded, $f(t,(1+$ $t) u, v$ ) is bounded on $J$.

$\left(\mathrm{H}_{2}\right) q(t)$ is a nonnegative measurable function defined in $J_{+}$and $q(t)$ does not identically vanish on any subinterval of $J_{+}$, and

$$
\begin{aligned}
& 0<\int_{0}^{+\infty} q(t) \mathrm{d} t<+\infty, \\
& 0<\int_{0}^{+\infty} \varphi_{p}^{-1}\left(\int_{s}^{+\infty} q(\tau) \mathrm{d} \tau\right) \mathrm{d} s<+\infty .
\end{aligned}
$$

$\left(\mathrm{H}_{3}\right) I_{k} \in C(J, J)$, and there exist $a_{k} \geq 0, b_{k} \geq 0$ such that

$$
\begin{aligned}
& 0 \leq I_{k}(x) \leq a_{k}+b_{k} x, \quad \text { for } x \in J(k=1,2,3, \ldots), \\
& a^{*}=\sum_{k=1}^{\infty} a_{k}<+\infty, \quad b^{*}=\sum_{k=1}^{\infty} b_{k}\left(1+t_{k}\right)<+\infty,
\end{aligned}
$$

with $b^{*}<(1 / 3)\left(1-\int_{0}^{+\infty} g(t) \mathrm{d} t\right)$.

If $p=2, I_{k}=0(k=1,2, \ldots), g(t) \equiv 0, x^{\prime}(\infty)=0$, then $\mathrm{BVP}(4)$ reduces to the following two-point boundary value problem:

$$
\begin{gathered}
-x^{\prime \prime}(t)=f\left(t, x(t), x^{\prime}(t)\right)=0, \quad t \in J, \\
x(0)=0, \quad x^{\prime}(\infty)=0
\end{gathered}
$$

which has been studied in [23].

Compared with $[8,21]$, the main features of the present paper are as follows. Firstly, second-order differential operator is replaced by a more general $p$-Laplacian operator. Secondly, in this paper, $x_{\infty}$ in boundary value conditions may not be zero which will bring about computational difficulties. Thirdly, by applying monotone iterative techniques, we construct successive iterative schemes starting off with simple known functions. It is worth pointing out that the first terms of our iterative schemes are simple functions. Therefore, the iterative schemes are significant and feasible.

The rest of this paper is organized as follows. In Section 2, we give some preliminaries and establish several lemmas. The main theorems are formulated and proved in Section 3. Then, in Section 4, an example is presented to illustrate the main results. 


\section{Preliminaries and Several Lemmas}

Definition 1. Let $E$ be a real Banach space. A nonempty closed set $P \subset E$ is said to be a cone provided that

(1) $a u+b v \in P$ for all $u, v \in P$ and all $a \geq 0, b \geq 0$,

(2) $u,-u \in P$ implies that $u=0$.

Definition 2. A map $\alpha: P \rightarrow[0,+\infty)$ is said to be concave on $P$, if $\alpha(t u+(1-t) v) \geq t \alpha(u)+(1-t) \alpha(v)$ for all $u, v \in P$ and $t \in[0,1]$.

Let $P C[J, R]=\{x: x$ is a map from $J$ into $R$ such that $x(t)$ is continuous at $t \neq t_{k}$, left continous at $t=t_{k}$ and $x\left(t_{k}^{+}\right)$ exists for $k=1,2, \ldots,\}, P C^{1}[J, R]=\left\{x \in P C[J, R]: x^{\prime}(t)\right.$ exists and is continuous at $t \neq t_{k}$, left continous at $t=t_{k}$ and $x^{\prime}\left(t_{k}^{+}\right)$exists for $\left.k=1,2, \ldots,\right\}$

$\operatorname{FPC}[J, R]$

$$
\begin{gathered}
=\left\{x \in P C[J, R]: \sup _{t \in J} \frac{|x(t)|}{1+t}<\infty\right\}, \\
E=D P C^{1}[J, R] \\
=\left\{x \in P C^{1}[J, R]: \sup _{t \in J} \frac{|x(t)|}{1+t}<\infty, \sup _{t \in J}\left|x^{\prime}(t)\right|<\infty\right\} .
\end{gathered}
$$

Obviously, $D P C^{1}[J, R] \subset F P C[J, R]$. It is clear that $F P C[J, R]$ is a Banach space with the norm

$$
\|x\|_{F}=\sup _{t \in J} \frac{|x(t)|}{1+t}
$$

and $D P C^{1}[J, R]$ is also a Banach space with the norm

$$
\|x\|_{D}=\max \left\{\|x\|_{F},\left\|x^{\prime}\right\|_{B}\right\}
$$

where $\left\|x^{\prime}\right\|_{B}=\sup _{t \in J}\left|x^{\prime}(t)\right|$. Let $J_{0}=\left[0, t_{1}\right], J_{k}=\left(t_{k}, t_{k+1}\right](k=$ $1,2,3, \ldots)$. Define a cone $P \subset E$ by

$$
P=\{x \in E: x \text { is concave and nondecreasing on } J \text {, }
$$

$$
\left.x(t) \geq 0, x^{\prime}(t) \geq 0, t \in J\right\} .
$$

Remark 3. If $x$ satisfies (4), then $\left(\varphi_{p}\left(x^{\prime}(t)\right)\right)^{\prime}=-q(t) f(t$, $\left.x(t), x^{\prime}(t)\right) \leq 0$, and $t \in[0,+\infty)$ which implies that $\varphi_{p}\left(x^{\prime}(t)\right)$ is nonincreasing on $J$; that is, $x^{\prime}(t)$ is also nonincreasing on $J$. Thus, $x$ is concave on $[0,+\infty)$. Moreover, if $x^{\prime}(\infty)=x_{\infty} \geq 0$, then $x^{\prime}(t) \geq 0, t \in[0,+\infty)$, and so $x$ is monotone increasing on $[0,+\infty)$.

Lemma 4. Let conditions $\left(H_{1}\right)-\left(H_{3}\right)$ hold. Then, $x \in P$ with $\left(\varphi_{p}\left(x^{\prime}(t)\right)\right)^{\prime} \in C(0,+\infty)$ is a solution of BVP (4) if and only if $x \in C[0,+\infty)$ is a fixed point of the following operator equation:

$(A x)(t)$

$$
\begin{aligned}
& =\frac{1}{1-\int_{0}^{+\infty} g(t) \mathrm{d} t} \\
& \times \int_{0}^{+\infty} g(t) \\
& \quad \times\left[\int_{0}^{t} \varphi_{p}^{-1}\right. \\
& \quad \times\left(\int_{s}^{+\infty} q(\tau) f\left(\tau, x(\tau), x^{\prime}(\tau)\right) \mathrm{d} \tau\right. \\
& \left.+\varphi_{p}\left(x_{\infty}\right)\right) \mathrm{d} s
\end{aligned}
$$

$$
\begin{gathered}
\left.+\sum_{t_{k}<t} I_{k}\left(x\left(t_{k}\right)\right)\right] \mathrm{d} t \\
+\int_{0}^{t} \varphi_{p}^{-1}\left(\int_{s}^{+\infty} q(\tau) f\left(\tau, x(\tau), x^{\prime}(\tau)\right) \mathrm{d} \tau\right. \\
\left.+\varphi_{p}\left(x_{\infty}\right)\right) \mathrm{d} s \\
+\sum_{t_{k}<t} I_{k}\left(x\left(t_{k}\right)\right) .
\end{gathered}
$$

Proof. Suppose that $x \in P$ with $\left(\varphi_{p}\left(x^{\prime}(t)\right)\right)^{\prime} \in C(0,+\infty)$ is a solution of BVP (4). For $t \in J$, integrating (4) from $t$ to $+\infty$, we have

$$
\begin{aligned}
& \int_{t}^{+\infty} \varphi_{p}\left(x^{\prime}(\tau)\right)^{\prime} \mathrm{d} \tau \\
& \quad=-\int_{t}^{+\infty} q(\tau) f\left(\tau, x(\tau), x^{\prime}(\tau)\right) \mathrm{d} \tau .
\end{aligned}
$$

That is

$$
\begin{aligned}
\varphi_{p}\left(x^{\prime}(\infty)\right)-\varphi_{p}\left(x^{\prime}(t)\right) \\
=-\int_{t}^{+\infty} q(\tau) f\left(\tau, x(\tau), x^{\prime}(\tau)\right) \mathrm{d} \tau,
\end{aligned}
$$

which implies that

$$
\begin{aligned}
& x^{\prime}(t) \\
& \quad=\varphi_{p}^{-1}\left(\int_{t}^{+\infty} q(\tau) f\left(\tau, x(\tau), x^{\prime}(\tau)\right) \mathrm{d} \tau+\varphi_{p}\left(x_{\infty}\right)\right) .
\end{aligned}
$$

If $t_{1}<t \leq t_{2}$, integrating (17) from 0 to $t_{1}$, we get that $x\left(t_{1}\right)-x(0)$

$$
=\int_{0}^{t_{1}} \varphi_{p}^{-1}\left(\int_{s}^{+\infty} q(\tau) f\left(\tau, x(\tau), x^{\prime}(\tau)\right) \mathrm{d} \tau+\varphi_{p}\left(x_{\infty}\right)\right) \mathrm{d} s .
$$


Integrating (17) from $t_{1}$ to $t$, we obtain

$$
\begin{aligned}
& x(t)-x\left(t_{1}^{+}\right) \\
& =\int_{t_{1}}^{t} \varphi_{p}^{-1}\left(\int_{s}^{+\infty} q(\tau) f\left(\tau, x(\tau), x^{\prime}(\tau)\right) \mathrm{d} \tau+\varphi_{p}\left(x_{\infty}\right)\right) \mathrm{d} s .
\end{aligned}
$$

Adding (18) and (19) together, we have

$$
\begin{aligned}
x(t) & \\
= & x(0) \\
& +\int_{0}^{t} \varphi_{p}^{-1}\left(\int_{s}^{+\infty} q(\tau) f\left(\tau, x(\tau), x^{\prime}(\tau)\right) \mathrm{d} \tau+\varphi_{p}\left(x_{\infty}\right)\right) \mathrm{d} s \\
& +I_{1}\left(x\left(t_{1}\right)\right), \quad t_{1}<t \leq t_{2} .
\end{aligned}
$$

Repeating previous process, we get that

$$
\begin{aligned}
x( & t) \\
= & x(0) \\
& +\int_{0}^{t} \varphi_{p}^{-1}\left(\int_{s}^{+\infty} q(\tau) f\left(\tau, x(\tau), x^{\prime}(\tau)\right) \mathrm{d} \tau+\varphi_{p}\left(x_{\infty}\right)\right) \mathrm{d} s \\
& +\sum_{t_{k}<t} I_{k}\left(x\left(t_{k}\right)\right) \\
= & \int_{0}^{+\infty} g(t) x(t) \mathrm{d} t \\
& +\int_{0}^{t} \varphi_{p}^{-1}\left(\int_{s}^{+\infty} q(\tau) f\left(\tau, x(\tau), x^{\prime}(\tau)\right) \mathrm{d} \tau+\varphi_{p}\left(x_{\infty}\right)\right) \mathrm{d} s \\
& +\sum_{t_{k}<t} I_{k}\left(x\left(t_{k}\right)\right) .
\end{aligned}
$$

It follows that

$$
\begin{gathered}
\int_{0}^{+\infty} g(t) x(t) \mathrm{d} t \\
=\frac{1}{1-\int_{0}^{+\infty} g(t) \mathrm{d} t} \\
\times \int_{0}^{+\infty} g(t) \\
\times\left[\int_{0}^{t} \varphi_{p}^{-1}+\int_{s}^{+\infty} q(\tau) f\left(\tau, x(\tau), x^{\prime}(\tau)\right) \mathrm{d} \tau\right. \\
\quad \times\left(\int_{p}\left(x_{\infty}\right)\right) \mathrm{d} s \\
\left.\quad+\sum_{t_{k}<t} I_{k}\left(x\left(t_{k}\right)\right)\right] \mathrm{d} t .
\end{gathered}
$$

Substituting (22) into (21), we get that

$$
\begin{aligned}
& x(t) \\
& =\frac{1}{1-\int_{0}^{+\infty} g(t) \mathrm{d} t} \\
& \times \int_{0}^{+\infty} g(t) \\
& \quad \times\left[\int_{0}^{t} \varphi_{p}^{-1}\right. \\
& \quad \times\left(\int_{s}^{+\infty} q(\tau) f\left(\tau, x(\tau), x^{\prime}(\tau)\right) \mathrm{d} \tau+\varphi_{p}\left(x_{\infty}\right)\right) \mathrm{d} s \\
& \left.\quad+\sum_{t_{k}<t} I_{k}\left(x\left(t_{k}\right)\right)\right] \mathrm{d} t \\
& +\int_{0}^{t} \varphi_{p}^{-1}\left(\int_{s}^{+\infty} q(\tau) f\left(\tau, x(\tau), x^{\prime}(\tau)\right) \mathrm{d} \tau+\varphi_{p}\left(x_{\infty}\right)\right) \mathrm{d} s \\
& \quad+\sum_{t_{k}<t} I_{k}\left(x\left(t_{k}\right)\right) .
\end{aligned}
$$

For $x \in P$, there exists $r_{0}$ such that $\|x\|_{D}<r_{0}$. Set $B_{r_{0}}=$ $\sup \left\{f(t,(1+t) u, v) \mid(t, u, v) \in J \times\left[0, r_{0}\right] \times\left[0, r_{0}\right]\right\}$, and we have by $\left(\mathrm{H}_{1}\right)$ and $\left(\mathrm{H}_{3}\right)$ that

$$
\begin{gathered}
\int_{0}^{+\infty} q(s) f\left(s, x(s), x^{\prime}(s)\right) \mathrm{d} s \leq \int_{0}^{+\infty} q(s) \mathrm{d} s \cdot B_{r_{0}}, \\
\sum_{t_{k}<t} I_{k}\left(x\left(t_{k}\right)\right) \leq \sum_{k=1}^{\infty} I_{k}\left(x\left(t_{k}\right)\right) \leq a^{*}+b^{*} r_{0}<+\infty .
\end{gathered}
$$

By (6), (24), we have

$$
\begin{aligned}
& x(t) \\
& =\frac{1}{1-\int_{0}^{+\infty} g(t) \mathrm{d} t} \\
& \times \int_{0}^{+\infty} g(t) \\
& \times\left[\int_{0}^{t} \varphi_{p}^{-1}\right. \\
& \quad \times\left(\int_{s}^{+\infty} q(\tau) f\left(\tau, x(\tau), x^{\prime}(\tau)\right) \mathrm{d} \tau\right. \\
& \left.+\sum_{t_{k}<t} I_{k}\left(x\left(t_{k}\right)\right)\right] \mathrm{d} t
\end{aligned}
$$




$$
\begin{aligned}
& +\int_{0}^{t} \varphi_{p}^{-1} \\
& \times\left(\int_{s}^{+\infty} q(\tau) f\left(\tau, x(\tau), x^{\prime}(\tau)\right) \mathrm{d} \tau+\varphi_{p}\left(x_{\infty}\right)\right) \mathrm{d} s \\
& +\sum_{t_{k}<t} I_{k}\left(x\left(t_{k}\right)\right) \\
& \leq \frac{1}{1-\int_{0}^{+\infty} g(t) \mathrm{d} t} \\
& \times \int_{0}^{+\infty} \operatorname{tg}(t) \mathrm{d} t \cdot \varphi_{p}^{-1} \\
& \times\left(\int_{0}^{+\infty} q(\tau) f\left(\tau, x(\tau), x^{\prime}(\tau)\right) \mathrm{d} \tau+\varphi_{p}\left(x_{\infty}\right)\right) \\
& +t \varphi_{p}^{-1}\left(\int_{0}^{+\infty} q(\tau) f\left(\tau, x(\tau), x^{\prime}(\tau)\right) \mathrm{d} \tau+\varphi_{p}\left(x_{\infty}\right)\right) \\
& +\frac{1}{1-\int_{0}^{+\infty} g(t) \mathrm{d} t}\left(a^{*}+b^{*} r_{0}\right) \\
& \leq 2^{1 /(p-1)}\left(\frac{1}{1-\int_{0}^{+\infty} g(t) \mathrm{d} t} \cdot \int_{0}^{+\infty} \operatorname{tg}(t) \mathrm{d} t+t\right) \\
& \times\left[\varphi_{p}^{-1}\left(\int_{0}^{+\infty} q(\tau) f\left(\tau, x(\tau), x^{\prime}(\tau)\right) \mathrm{d} \tau\right)+x_{\infty}\right] \\
& +\frac{1}{1-\int_{0}^{+\infty} g(t) \mathrm{d} t}\left(a^{*}+b^{*} r_{0}\right) \text {. }
\end{aligned}
$$

It follows from (24) and (25) that the right term in (23) is well defined. Thus, we have proved that $x$ is a fixed point of the operator $A$ defined by (14).

Conversely, suppose that $x \in C[0,+\infty)$ is a fixed point of the operator equation (14). Evidently,

$$
\left.\Delta x\right|_{t=t_{k}}=I_{k}\left(x\left(t_{k}\right)\right) \quad(k=1,2, \ldots) .
$$

Direct differentiation of (14) implies that, for $t \neq t_{k}$,

$$
\begin{gathered}
x^{\prime}(t)=\varphi_{p}^{-1}\left(\int_{t}^{+\infty} q(s) f\left(s, x(s), x^{\prime}(s)\right) \mathrm{d} s+\varphi_{p}\left(x_{\infty}\right)\right) \\
\left.\Delta x^{\prime}\right|_{t=t_{k}}=0 \quad(k=1,2, \ldots) \\
\left(\varphi_{p}\left(x^{\prime}(t)\right)\right)^{\prime}=-q(t) f\left(t, x(t), x^{\prime}(t)\right)
\end{gathered}
$$

which means that $\left(\varphi_{p}\left(x^{\prime}(t)\right)\right)^{\prime} \in C\left(J^{\prime}\right)$. It is easy to verify that $x(0)=\int_{0}^{+\infty} g(t) x(t) \mathrm{d} t, x^{\prime}(\infty)=x_{\infty}$. The proof of Lemma 4 is complete.

To obtain the complete continuity of $A$, the following lemma is still needed.
Lemma 5 (see $[33,34])$. Let $W$ be a bounded subset of $P$. Then, $P$ is relatively compact in $E$ if $\{W(t) /(1+t)\}$ and $\left\{W^{\prime}(t)\right\}$ are both equicontinuous on any finite subinterval $J_{k} \cap[0, T](k=$ $1,2, \ldots)$ for any $T>0$, and for any $\varepsilon>0$, there exists $N>0$ such that

$$
\left|\frac{x\left(t^{\prime}\right)}{1+t^{\prime}}-\frac{x\left(t^{\prime \prime}\right)}{1+t^{\prime \prime}}\right|<\varepsilon, \quad\left|x^{\prime}\left(t^{\prime}\right)-x^{\prime}\left(t^{\prime \prime}\right)\right|<\varepsilon, \quad \forall t^{\prime}, t^{\prime \prime} \geq N,
$$

uniformly with respect to $x \in W$ as $t^{\prime}, t^{\prime \prime} \geq N$, where $W(t)=$ $\{x(t) \mid x \in W\}, W^{\prime}(t)=\left\{x^{\prime}(t) \mid x \in W\right\}, t \in[0,+\infty)$.

This lemma is a simple improvement of the Corduneanu theorem in $[33,34]$.

Lemma 6. Let $\left(H_{1}\right)-\left(H_{3}\right)$ hold. Then $A: P \rightarrow P$ is completely continuous.

Proof. For any $x \in P$, by (14), we have

$$
\begin{gathered}
\varphi_{p}\left((A x)^{\prime}\right)(t)=\int_{t}^{+\infty} q(s) f\left(s, x(s), x^{\prime}(s)\right) \mathrm{d} s+\varphi_{p}\left(x_{\infty}\right), \\
\left(\varphi_{p}(A x)^{\prime}(t)\right)^{\prime}=-q(t) f\left(t, x(t), x^{\prime}(t)\right) .
\end{gathered}
$$

It follows from (14), (29), and $\left(\mathrm{H}_{1}\right)$ that $(A x)(t) \geq 0,(A x)^{\prime}(t) \geq$ $x_{\infty} \geq 0,(A x)^{\prime \prime}(t) \leq 0$, that is, $A(P) \subset P$. Now, we prove that $A$ is continuous and compact respectively. Let $x_{n} \in P, x_{n} \rightarrow x$ as $n \rightarrow \infty$, then there exists $r_{0}$ such that $\sup _{n \in N \backslash\{0\}}\left\|x_{n}\right\|<r_{0}$. Let $B_{r_{0}}=\sup \left\{f(t,(1+t) u, v) \mid(t, u, v) \in J \times\left[0, r_{0}\right] \times\left[0, r_{0}\right]\right\}$. By $\left(\mathrm{H}_{1}\right)$ and $\left(\mathrm{H}_{2}\right)$, we have

$$
\begin{aligned}
& \int_{0}^{+\infty} \quad q(\tau)\left|f\left(\tau, x_{n}(\tau), x_{n}^{\prime}(\tau)\right)-f\left(\tau, x(\tau), x^{\prime}(\tau)\right)\right| \mathrm{d} \tau \\
& \quad \leq 2 B_{r_{0}} \cdot \int_{0}^{+\infty} q(s) \mathrm{d} s<+\infty .
\end{aligned}
$$

It follows from (30) and dominated convergence theorem that

$$
\begin{aligned}
& \int_{0}^{+\infty} q(\tau)\left|f\left(\tau, x_{n}(\tau), x_{n}^{\prime}(\tau)\right)-f\left(\tau, x(\tau), x^{\prime}(\tau)\right)\right| \mathrm{d} \tau \\
& \longrightarrow \int_{0}^{+\infty} q(\tau)\left|f\left(\tau, x(\tau), x^{\prime}(\tau)\right)-f\left(\tau, x(\tau), x^{\prime}(\tau)\right)\right| \mathrm{d} \tau,
\end{aligned}
$$

which implies that

$$
\begin{aligned}
& \mid \varphi_{p}^{-1}\left(\int_{0}^{+\infty} q(\tau) f\left(\tau, x_{n}(\tau), x_{n}^{\prime}(\tau)\right) \mathrm{d} \tau+\varphi_{p}\left(x_{\infty}\right)\right) \mathrm{d} s \\
& -\varphi_{p}^{-1}\left(\int_{0}^{+\infty} q(\tau) f\left(\tau, x(\tau), x^{\prime}(\tau)\right) \mathrm{d} \tau+\varphi_{p}\left(x_{\infty}\right)\right) \mathrm{d} s \mid \\
& \longrightarrow 0, \quad n \longrightarrow \infty .
\end{aligned}
$$


By (30)-(32), $\left(\mathrm{H}_{3}\right)$ and dominated convergence theorem, we get that

$$
\begin{aligned}
& \left\|A x_{n}-A x\right\|_{F} \\
& =\sup _{t \in J}\left\{\frac{1}{1+t}\right. \\
& \times \mid \frac{1}{1-\int_{0}^{+\infty} g(t) \mathrm{d} t} \\
& \times\left[\int_{0}^{+\infty} g(t)\right. \\
& \times \int_{0}^{t} \varphi_{p}^{-1} \\
& \times\left(\int_{s}^{+\infty} q(\tau) f\left(\tau, x_{n}(\tau), x_{n}^{\prime}(\tau)\right) \mathrm{d} \tau\right. \\
& \left.+\varphi_{p}\left(x_{\infty}\right)\right) \mathrm{d} s \mathrm{~d} t \\
& -\int_{0}^{+\infty} g(t) \\
& \times \int_{0}^{t} \varphi_{p}^{-1}\left(\int_{s}^{+\infty} q(\tau) f\left(\tau, x(\tau), x^{\prime}(\tau)\right) \mathrm{d} \tau\right. \\
& +\int_{0}^{+\infty} g(t) \\
& \left.+\varphi_{p}\left(x_{\infty}\right)\right) \mathrm{d} s \mathrm{~d} t \\
& \left.\cdot \sum_{t_{k}<t}\left(I_{k}\left(x_{n}\left(t_{k}\right)\right)-I_{k}\left(x\left(t_{k}\right)\right)\right)\right] \mathrm{d} t \\
& +\int_{0}^{t}\left[\varphi _ { p } ^ { - 1 } \left(\int_{s}^{+\infty} q(\tau) f\left(\tau, x_{n}(\tau), x_{n}^{\prime}(\tau)\right) \mathrm{d} \tau\right.\right. \\
& \left.+\varphi_{p}\left(x_{\infty}\right)\right) \\
& -\varphi_{p}^{-1}\left(\int_{s}^{+\infty} q(\tau) f\left(\tau, x(\tau), x^{\prime}(\tau)\right) \mathrm{d} \tau\right. \\
& \left.\left.+\varphi_{p}\left(x_{\infty}\right)\right)\right] \mathrm{d} s \\
& \left.+\sum_{t_{k}<t}\left(I_{k}\left(x_{n}\left(t_{k}\right)\right)-I_{k}\left(x\left(t_{k}\right)\right)\right) \mid\right\} \\
& \leq \frac{1}{1-\int_{0}^{+\infty} g(t) \mathrm{d} t} \\
& \times \int_{0}^{+\infty} \operatorname{tg}(t) \mathrm{d} t \\
& \cdot \mid \varphi_{p}^{-1}\left(\int_{0}^{+\infty} q(\tau) f\left(\tau, x_{n}(\tau), x_{n}^{\prime}(\tau)\right) \mathrm{d} \tau+\varphi_{p}\left(x_{\infty}\right)\right) \\
& -\varphi_{p}^{-1}\left(\int_{0}^{+\infty} q(\tau) f\left(\tau, x(\tau), x^{\prime}(\tau)\right) \mathrm{d} \tau+\varphi_{p}\left(x_{\infty}\right)\right)
\end{aligned}
$$

$$
\begin{aligned}
& +\mid \varphi_{p}^{-1}\left(\int_{0}^{+\infty} q(\tau) f\left(\tau, x_{n}(\tau), x_{n}^{\prime}(\tau)\right) \mathrm{d} \tau+\varphi_{p}\left(x_{\infty}\right)\right) \\
& \quad-\varphi_{p}^{-1}\left(\int_{0}^{+\infty} q(\tau) f\left(\tau, x(\tau), x^{\prime}(\tau)\right) \mathrm{d} \tau+\varphi_{p}\left(x_{\infty}\right)\right) \mid \\
& +\frac{1}{1-\int_{0}^{+\infty} g(t) \mathrm{d} t} \\
& \times \int_{0}^{+\infty} g(t) \cdot \sum_{t_{k}<t}\left|I_{k}\left(x_{n}\left(t_{k}\right)\right)-I_{k}\left(x\left(t_{k}\right)\right)\right| \mathrm{d} t \\
& +\sum_{t_{k}<t}\left|I_{k}\left(x_{n}\left(t_{k}\right)\right)-I_{k}\left(x\left(t_{k}\right)\right)\right| \longrightarrow 0 \quad(n \longrightarrow \infty), \\
& \left\|\left(A x_{n}\right)^{\prime}-(A x)^{\prime}\right\|_{B} \\
& =\sup _{t \in J}\left\{\mid \varphi_{p}^{-1}\left(\int_{t}^{+\infty} q(s) f\left(s, x_{n}(s), x_{n}^{\prime}(s)\right) \mathrm{d} s+\varphi_{p}\left(x_{\infty}\right)\right)\right. \\
& \left.\quad-\varphi_{p}^{-1}\left(\int_{t}^{+\infty} q(s) f\left(s, x(s), x^{\prime}(s)\right) \mathrm{d} s+\varphi_{p}\left(x_{\infty}\right)\right) \mid\right\} \\
& \longrightarrow 0 \quad(n \longrightarrow \infty) .
\end{aligned}
$$

It follows from (33) that $A$ is continuous.

Let $\Omega \subset P$ be any bounded subset. Then, there exists $r>0$ such that $\|x\|_{D} \leq r$ for any $x \in \Omega$. Obviously,

$\|A x\|_{F}$

$$
\begin{aligned}
& =\sup _{t \in J}\left\{\frac{1}{1+t}\right. \\
& \times \mid \frac{1}{1-\int_{0}^{+\infty} g(t) \mathrm{d} t} \\
& \times \int_{0}^{+\infty} g(t) \\
& \times\left[\int_{0}^{t} \varphi_{p}^{-1}\right. \\
& \times\left(\int_{s}^{+\infty} q(\tau) f\left(\tau, x(\tau), x^{\prime}(\tau)\right) \mathrm{d} \tau\right. \\
& \left.+\varphi_{p}\left(x_{\infty}\right)\right) \mathrm{d} s \\
& \left.+\sum_{t_{k}<t} I_{k}\left(x\left(t_{k}\right)\right)\right] \mathrm{d} t \\
& +\int_{0}^{t} \varphi_{p}^{-1} \\
& \times\left(\int_{s}^{+\infty} q(\tau) f\left(\tau, x(\tau), x^{\prime}(\tau)\right) \mathrm{d} \tau\right. \\
& \left.+\varphi_{p}\left(x_{\infty}\right)\right) \mathrm{d} s \\
& \left.+\sum_{t_{k}<t} I_{k}\left(x\left(t_{k}\right)\right) \mid\right\}
\end{aligned}
$$




$$
\begin{aligned}
\leq & \frac{1}{1-\int_{0}^{+\infty} g(t) \mathrm{d} t} \\
& \times \int_{0}^{+\infty} \operatorname{tg}(t) \mathrm{d} t \cdot \varphi_{p}^{-1} \\
& \times\left(\int_{0}^{+\infty} q(\tau) f\left(\tau, x(\tau), x^{\prime}(\tau)\right) \mathrm{d} \tau+\varphi_{p}\left(x_{\infty}\right)\right) \\
& +\varphi_{p}^{-1}\left(\int_{0}^{+\infty} q(\tau) f\left(\tau, x(\tau), x^{\prime}(\tau)\right) \mathrm{d} \tau+\varphi_{p}\left(x_{\infty}\right)\right) \\
& +\frac{1-\int_{0}^{+\infty} g(t) \mathrm{d} t}{1}\left(a^{*}+b^{*} r\right) \\
\leq & 2^{1 /(p-1)}\left[\frac{1}{1-\int_{0}^{+\infty} g(t) \mathrm{d} t} \int_{0}^{+\infty} t g(t) \mathrm{d} t+1\right] \\
\leq & \times\left[\varphi_{p}^{-1 /(p-1)}\left[\varphi_{p}^{-1}\left(\int_{r}^{+\infty} q(s) \mathrm{d} s\right) \cdot \varphi_{p}^{-1}\left(\int_{0}^{+\infty} q(\tau) \mathrm{d} \tau\right)+x_{\infty}\right]\right. \\
& +\frac{1}{1-\int_{0}^{+\infty} g(t) \mathrm{d} t}\left(a^{*}+b^{*} r\right) \\
\left\|(A x)^{\prime}\right\|_{B} & \sup \left\{\mid \varphi_{p}^{-1}\left(\int_{t}^{+\infty} q(s) f\left(s, x(s), x^{\prime}(s)\right) \mathrm{d} s+\varphi_{p}\left(x_{\infty}\right)\right)\right\}
\end{aligned}
$$

From (34), $\left(\mathrm{H}_{2}\right)$, and $\left(\mathrm{H}_{3}\right)$, we know that $A \Omega$ is bounded.

For any $T>0, x \in \Omega, t^{\prime}, t^{\prime \prime} \in J_{k} \cap[0, T]$ with $t^{\prime}<t^{\prime \prime}$, by the absolute continuity of the integral, we have

$$
\begin{aligned}
& \left|\frac{(A x)\left(t^{\prime}\right)}{1+t^{\prime}}-\frac{(A x)\left(t^{\prime \prime}\right)}{1+t^{\prime \prime}}\right| \\
& \leq \frac{1}{\left(1+t^{\prime \prime}\right)\left(1-\int_{0}^{+\infty} g(t) \mathrm{d} t\right)} \\
& \quad \cdot \int_{0}^{+\infty} g(t) \mathrm{d} t \\
& \quad \cdot \int_{t^{\prime}}^{t^{\prime \prime}} \varphi_{p}^{-1} \\
& \quad \times\left(\int_{s}^{+\infty} q(\tau) f\left(\tau, x(\tau), x^{\prime}(\tau)\right) \mathrm{d} \tau+\varphi_{p}\left(x_{\infty}\right)\right) \mathrm{d} s \\
& \quad+\frac{1}{1-\int_{0}^{+\infty} g(t) \mathrm{d} t} \\
& \quad \times \int_{0}^{+\infty} g(t) \mathrm{d} t
\end{aligned}
$$

$$
\begin{aligned}
& \int_{0}^{t^{\prime}} \varphi_{p}^{-1} \\
& \times\left(\int_{s}^{+\infty} q(\tau) f\left(\tau, x(\tau), x^{\prime}(\tau)\right) \mathrm{d} \tau+\varphi_{p}\left(x_{\infty}\right)\right) \mathrm{d} s \\
& \cdot\left|\frac{1}{1+t^{\prime \prime}}-\frac{1}{1+t^{\prime}}\right| \\
& +\frac{1}{1+t^{\prime \prime}} \\
& \times \int_{t^{\prime}}^{t^{\prime \prime}} \varphi_{p}^{-1} \\
& \times\left(\int_{s}^{+\infty} q(\tau) f\left(\tau, x(\tau), x^{\prime}(\tau)\right) \mathrm{d} \tau+\varphi_{p}\left(x_{\infty}\right)\right) \mathrm{d} s \\
& +\int_{0}^{t^{\prime}} \varphi_{p}^{-1} \\
& \times\left(\int_{s}^{+\infty} q(\tau) f\left(\tau, x(\tau), x^{\prime}(\tau)\right) \mathrm{d} \tau+\varphi_{p}\left(x_{\infty}\right)\right) \mathrm{d} s \\
& \cdot\left|\frac{1}{1+t^{\prime \prime}}-\frac{1}{1+t^{\prime}}\right| \\
& +\frac{a^{*}+b^{*} r}{1-\int_{0}^{+\infty} g(t) \mathrm{d} t} \cdot\left|\frac{1}{1+t^{\prime \prime}}-\frac{1}{1+t^{\prime}}\right| \\
& \leq \frac{2^{1 /(p-1)}}{1-\int_{0}^{+\infty} g(t) \mathrm{d} t} \\
& \times\left[\int_{t^{\prime}}^{t^{\prime \prime}}\left(\varphi_{p}^{-1}\left(\int_{0}^{+\infty} q(\tau) \mathrm{d} \tau\right) \cdot \varphi_{p}^{-1}\left(B_{r}\right)+x_{\infty}\right) \mathrm{d} s\right. \\
& +\int_{0}^{t^{\prime}}\left(\varphi_{p}^{-1}\left(\int_{0}^{+\infty} q(\tau) \mathrm{d} \tau\right) \cdot \varphi_{p}^{-1}\left(B_{r}\right)+x_{\infty}\right) \mathrm{d} s \\
& \left.\cdot\left|\frac{1}{1+t^{\prime \prime}}-\frac{1}{1+t^{\prime}}\right|\right] \\
& +\frac{a^{*}+b^{*} r}{1-\int_{0}^{+\infty} g(t) \mathrm{d} t} \cdot\left|\frac{1}{1+t^{\prime \prime}}-\frac{1}{1+t^{\prime}}\right| \\
& \longrightarrow 0 \text { uniformly as } t^{\prime} \longrightarrow t^{\prime \prime} \text {, } \\
& \left|\varphi_{p}\left((A x)^{\prime}\left(t^{\prime}\right)\right)-\varphi_{p}\left((A x)^{\prime}\left(t^{\prime \prime}\right)\right)\right| \\
& =\left|\int_{t^{\prime}}^{t^{\prime \prime}} q(s) f\left(s, x(s), x^{\prime}(s)\right) \mathrm{d} s\right| \\
& \leq B_{r} \cdot\left|\int_{t^{\prime}}^{t^{\prime \prime}} q(s) \mathrm{d} s\right| \\
& \longrightarrow 0 \text { uniformly as } t^{\prime} \longrightarrow t^{\prime \prime} \text {. }
\end{aligned}
$$

Thus, we have proved that $A \Omega$ is equicontinuous on any $J_{k} \cap$ $[0, T]$. 
Next, we prove that for any $\varepsilon>0, x \in \Omega$, there exits sufficiently large $N>0$ such that

$$
\begin{aligned}
& \left|\frac{(A x)\left(t^{\prime}\right)}{1+t^{\prime}}-\frac{(A x)\left(t^{\prime \prime}\right)}{1+t^{\prime \prime}}\right|<\varepsilon, \\
& \left|(A x)^{\prime}\left(t^{\prime}\right)-(A x)^{\prime}\left(t^{\prime \prime}\right)\right|<\varepsilon, \quad \forall t^{\prime}, t^{\prime \prime} \geq N .
\end{aligned}
$$

For any $x \in \Omega$, we have

$$
\begin{aligned}
& \lim _{t \rightarrow+\infty} \frac{1}{1+t} \\
& {\left[\frac{1}{1-\int_{0}^{+\infty} g(t) \mathrm{d} t}\right.} \\
& \left.\times \int_{0}^{+\infty} g(t) \sum_{t_{k}<t} I_{k}\left(x\left(t_{k}\right)\right) \mathrm{d} t+\sum_{t_{k}<t} I_{k}\left(x\left(t_{k}\right)\right)\right] \\
& \leq \lim _{t \rightarrow+\infty} \frac{1}{1+t} \cdot \frac{a^{*}+b^{*} r}{1-\int_{0}^{+\infty} g(t) \mathrm{d} t}=0, \\
& \lim _{t \rightarrow+\infty} \frac{1}{1+t} \\
& \cdot \int_{0}^{+\infty} g(t) \\
& \cdot\left[\int _ { 0 } ^ { t } \varphi _ { p } ^ { - 1 } \left(\int_{s}^{+\infty} q(\tau) f\left(\tau, x(\tau), x^{\prime}(\tau)\right) \mathrm{d} \tau\right.\right. \\
& \left.\left.+\varphi_{p}\left(x_{\infty}\right)\right) \mathrm{d} s\right] \mathrm{d} t \\
& \leq \lim _{t \rightarrow+\infty} \frac{1}{1+t} 2^{1 /(p-1)} \\
& \times\left(\frac{1}{1-\int_{0}^{+\infty} g(t) \mathrm{d} t} \int_{0}^{+\infty} \operatorname{tg}(t) \mathrm{d} t\right) \\
& \times\left[\varphi_{p}^{-1}\left(B_{r}\right) \cdot \varphi_{p}^{-1}\left(\int_{0}^{+\infty} q(\tau) \mathrm{d} \tau\right)+x_{\infty}\right]=0, \\
& \lim _{t \rightarrow+\infty} \frac{1}{1+t} \\
& \cdot \int_{0}^{t} \varphi_{p}^{-1}\left(\int_{s}^{+\infty} q(\tau) f\left(\tau, x(\tau), x^{\prime}(\tau)\right) \mathrm{d} \tau+\varphi_{p}\left(x_{\infty}\right)\right) \mathrm{d} s \\
& =\lim _{t \rightarrow+\infty} \varphi_{p}^{-1}\left(\int_{t}^{+\infty} q(\tau) f\left(\tau, x(\tau), x^{\prime}(\tau)\right) \mathrm{d} \tau+\varphi_{p}\left(x_{\infty}\right)\right) \\
& =x_{\infty} \text {. }
\end{aligned}
$$

It follows from (37) that

$$
\begin{aligned}
& \lim _{t \rightarrow \infty}\left|\frac{(A x)(t)}{1+t}\right| \\
& =\lim _{t \rightarrow \infty} \frac{1}{1+t} \\
& \times\left\{\frac{1}{1-\int_{0}^{+\infty} g(t) d t}\right. \\
& \times \int_{0}^{+\infty} g(t) \\
& \times\left[\int_{0}^{t} \varphi_{p}^{-1}\right. \\
& \times\left(\int_{s}^{+\infty} q(\tau) f\left(\tau, x(\tau), x^{\prime}(\tau)\right) \mathrm{d} \tau\right. \\
& \left.+\varphi_{p}\left(x_{\infty}\right)\right) \mathrm{d} s \\
& \left.+\sum_{t_{k}<t} I_{k}\left(x\left(t_{k}\right)\right)\right] \mathrm{d} t \\
& +\int_{0}^{t} \varphi_{p}^{-1} \\
& \times\left(\int_{s}^{+\infty} q(\tau) f\left(\tau, x(\tau), x^{\prime}(\tau)\right) \mathrm{d} \tau\right. \\
& \left.+\varphi_{p}\left(x_{\infty}\right)\right) \mathrm{d} s \\
& \left.+\sum_{t_{k}<t} I_{k}\left(x\left(t_{k}\right)\right)\right\} \\
& =x_{\infty} \text {. }
\end{aligned}
$$

On the other hand, we arrive at

$$
\begin{aligned}
& \lim _{t \rightarrow \infty}\left|(A x)^{\prime}(t)\right| \\
& \quad=\lim _{t \rightarrow \infty} \varphi_{p}^{-1}\left(\int_{t}^{+\infty} q(s) f\left(s, x(s), x^{\prime}(s)\right) \mathrm{d} s+\varphi_{p}\left(x_{\infty}\right)\right) \\
& \quad=x_{\infty} .
\end{aligned}
$$

Thus, (36) can be easily derived from (38) and (39). So, by Lemma 5, we know that $A \Omega$ is relatively compact. Thus, we have proved that $A: P \rightarrow P$ is completely continuous. 


\section{Main Results}

For notational convenience, we denote that

$$
\begin{aligned}
& m=2^{1 /(p-1)}\left(\frac{1}{1-\int_{0}^{+\infty} g(t) \mathrm{d} t} \int_{0}^{+\infty} \operatorname{tg}(t) \mathrm{d} t+1\right) \\
& \cdot \varphi_{p}^{-1}\left(\int_{0}^{+\infty} q(\tau) \mathrm{d} \tau\right) \\
& m^{\prime}=\left(\frac{1}{1-\int_{0}^{+\infty} g(t) \mathrm{d} t} \int_{0}^{+\infty} \operatorname{tg}(t) \mathrm{d} t+1\right) \\
& \cdot \varphi_{p}^{-1}\left(\int_{0}^{+\infty} q(\tau) \mathrm{d} \tau\right) \\
& n=2^{1 /(p-1)}\left(\frac{1}{1-\int_{0}^{+\infty} g(t) \mathrm{d} t} \int_{0}^{+\infty} \operatorname{tg}(t) \mathrm{d} t+1\right) x_{\infty} \\
& n^{\prime}=\left(\frac{1}{1-\int_{0}^{+\infty} g(t) \mathrm{d} t} \int_{0}^{+\infty} \operatorname{tg}(t) \mathrm{d} t+1\right) x_{\infty}, \\
& \Lambda=\max \left\{\frac{a^{*}}{1-\int_{0}^{+\infty} g(t) \mathrm{d} t-3 b^{*}}, n\right\}, \\
& \Lambda^{\prime}=\max \left\{\frac{a^{*}}{1-\int_{0}^{+\infty} g(t) \mathrm{d} t-3 b^{*}}, n^{\prime}\right\} \text {. }
\end{aligned}
$$

Theorem 7. Assume that $\left(H_{1}\right)-\left(H_{3}\right)$ hold, and there exists

$$
d> \begin{cases}3 \Lambda, & \text { as } p \geq 2, \\ 3 \Lambda^{\prime}, & \text { as } 1<p<2\end{cases}
$$

such that

$\left(\mathrm{A}_{1}\right) f\left(t, x_{1}, y_{1}\right) \leq f\left(t, x_{2}, y_{2}\right)$ for any $0 \leq t<+\infty, 0 \leq$ $x_{1} \leq x_{2} \leq d, 0 \leq y_{1} \leq y_{2} \leq d$,

$\left(\mathrm{A}_{2}\right)$

$$
\begin{aligned}
& f(t,(1+t) u, v) \leq \begin{cases}\varphi_{p}\left(\frac{d}{3 m}\right), & \text { as } p \geq 2, \\
\varphi_{p}\left(\frac{d}{3 m^{\prime}}\right), & \text { as } 1<p<2,\end{cases} \\
& (t, u, v) \in[0,+\infty) \times[0, d] \times[0, d],
\end{aligned}
$$

$\left(\mathrm{A}_{3}\right) I_{k}\left(x_{1}\right) \leq I_{k}\left(x_{2}\right)(k=1,2, \ldots$,$) , for any 0 \leq x_{1} \leq x_{2}$.

Then, the boundary value problem (4) admits positive, nondecreasing on $[0,+\infty)$ and concave solutions $w^{*}$ and $v^{*}$ such that $0<\left\|w^{*}\right\|_{D} \leq d$, and $\lim _{n \rightarrow \infty} w_{n}=\lim _{n \rightarrow \infty} A^{n} w_{0}=$ $w^{*}$, where

$$
w_{0}(t)=d+d t, \quad t \in J,
$$

and $0<\left\|v^{*}\right\|_{D} \leq d, \lim _{n \rightarrow \infty} v_{n}=\lim _{n \rightarrow \infty} A^{n} v_{0}=v^{*}$, where $v_{0}(t)=0, t \in J$.

Proof. We only prove the case that $p \geq 2$. Another case can be proved in a similar way. By Lemma 6 , we know that $A: P \rightarrow$ $P$ is completely continuous. From the definition of $A,\left(\mathrm{~A}_{1}\right)$, and $\left(\mathrm{A}_{3}\right)$, we can easily get that $A x_{1} \leq A x_{2}$ for any $x_{1}, x_{2} \in P$ with $x_{1} \leq x_{2}, x_{1}^{\prime} \leq x_{2}^{\prime}$. Denote that

$$
\bar{P}_{d}=\left\{x \in P \mid\|x\|_{D} \leq d\right\} .
$$

In what follows, we first prove that $A: \bar{P}_{d} \rightarrow \bar{P}_{d}$. If $x \in \bar{P}_{d}$, then $\|x\|_{D} \leq d$. By (6), (40), (42), (44), $\left(\mathrm{H}_{3}\right),\left(\mathrm{A}_{2}\right)$, and $\left(\mathrm{A}_{3}\right)$, we get that

$\|A x\|_{F}$

$$
\begin{aligned}
=\sup _{t \in J}\left\{\frac{1}{1+t}\right. & \\
\times & \mid \frac{1}{1-\int_{0}^{+\infty} g(t) \mathrm{d} t} \\
& \times \int_{0}^{+\infty} g(t) \\
& \times\left[\int_{0}^{t} \varphi_{p}^{-1}\right.
\end{aligned}
$$$$
\times\left(\int_{s}^{+\infty} q(\tau) f\left(\tau, x(\tau), x^{\prime}(\tau)\right) \mathrm{d} \tau\right.
$$$$
\left.+\varphi_{p}\left(x_{\infty}\right)\right) \mathrm{d} s
$$

$$
\left.+\sum_{t_{k}<t} I_{k}\left(x\left(t_{k}\right)\right)\right] \mathrm{d} t
$$

$$
+\int_{0}^{t} \varphi_{p}^{-1}
$$$$
\times\left(\int_{s}^{+\infty} q(\tau) f\left(\tau, x(\tau), x^{\prime}(\tau)\right) \mathrm{d} \tau\right.
$$$$
\left.+\varphi_{p}\left(x_{\infty}\right)\right) \mathrm{d} s
$$

$$
\left.+\sum_{t_{k}<t} I_{k}\left(x\left(t_{k}\right)\right) \mid\right\}
$$

$$
\begin{aligned}
& \leq \frac{1}{1-\int_{0}^{+\infty} g(t) \mathrm{d} t} \\
& \quad \times \int_{0}^{+\infty} \operatorname{tg}(t) \mathrm{d} t \cdot \varphi_{p}^{-1} \\
& \quad \times\left(\int_{0}^{+\infty} q(\tau) f\left(\tau, x(\tau), x^{\prime}(\tau)\right) \mathrm{d} \tau+\varphi_{p}\left(x_{\infty}\right)\right)
\end{aligned}
$$




$$
\begin{aligned}
& \quad+\varphi_{p}^{-1}\left(\int_{0}^{+\infty} q(\tau) f\left(\tau, x(\tau), x^{\prime}(\tau)\right) \mathrm{d} \tau+\varphi_{p}\left(x_{\infty}\right)\right) \\
& +\frac{1}{1-\int_{0}^{+\infty} g(t) \mathrm{d} t}\left(a^{*}+b^{*} d\right) \\
& \leq 2^{1 /(p-1)}\left[\frac{1}{1-\int_{0}^{+\infty} g(t) \mathrm{d} t} \int_{0}^{+\infty} t g(t) \mathrm{d} t+1\right] \\
& \quad \times\left[\varphi_{p}^{-1}\left(\varphi_{p}\left(\frac{d}{3 m}\right)\right) \cdot \varphi_{p}^{-1}\left(\int_{0}^{+\infty} q(\tau) \mathrm{d} \tau\right)+x_{\infty}\right] \\
& \quad+\frac{1}{1-\int_{0}^{+\infty} g(t) \mathrm{d} t}\left(a^{*}+b^{*} d\right) \\
& \leq \frac{d}{3}+\frac{d}{3}+\frac{d}{3}=d, \\
& \left\|(A x)^{\prime}\right\|_{B} \\
& \leq 2^{1 /(p-1)}\left[\varphi_{p}^{-1}\left(\int_{0}^{+\infty} q(s) \mathrm{d} s\right) \varphi_{p}^{-1}\left(\varphi_{p}\left(\frac{d}{3 m}\right)\right)+x_{\infty}\right] \\
& \quad \sup _{t \in J}\left\{\varphi_{p}^{-1}\left(\int_{t}^{+\infty} q(s) f\left(s, x(s), x^{\prime}(s)\right) \mathrm{d} s+\varphi_{p}\left(x_{\infty}\right)\right) \mid\right\}
\end{aligned}
$$

Thus, we get that $\|A x\|_{D} \leq d$. Hence, we have proved that $A: \bar{P}_{d} \rightarrow \bar{P}_{d}$.

Let $w_{0}(t)=d+d t, 0 \leq t<+\infty$, then $w_{0}(t) \in \bar{P}_{d}$. Let $w_{1}=A w_{0}, w_{2}=A^{2} w_{0}$, then by Lemma 6 , we have that $w_{1} \in$ $\bar{P}_{d}$ and $w_{2} \in \bar{P}_{d}$. Denote that

$$
w_{n+1}=A w_{n}=A^{n+1} w_{0}, \quad n=0,1,2, \ldots
$$

Since $A: \bar{P}_{d} \rightarrow \bar{P}_{d}$, we have that

$$
w_{n} \in A\left(\bar{P}_{d}\right) \subset \bar{P}_{d}, n=1,2,3, \ldots
$$

It follows from the complete continuity of $A$ that $\left\{w_{n}\right\}_{n=1}^{\infty}$ is a sequentially compact set. We assert that $\left\{w_{n}\right\}_{n=1}^{\infty}$ has a convergent subsequence $\left\{w_{n_{k}}\right\}_{k=1}^{\infty}$, and there exists $w^{*} \in \bar{P}_{d}$ such that $w_{n_{k}} \rightarrow w^{*}$.

By $(51),\left(\mathrm{A}_{1}\right)-\left(\mathrm{A}_{3}\right)$, we get that

$$
w_{1}(t)
$$

$$
\begin{gathered}
=\frac{1}{1-\int_{0}^{+\infty} g(t) \mathrm{d} t} \\
\times \int_{0}^{+\infty} g(t) \\
\times\left[\int_{0}^{t} \varphi_{p}^{-1}\right.
\end{gathered}
$$

$$
\begin{gathered}
\times\left(\int_{s}^{+\infty} q(\tau) f\left(\tau, w_{0}(\tau), w_{0}^{\prime}(\tau)\right) \mathrm{d} \tau\right. \\
\left.+\varphi_{p}\left(x_{\infty}\right)\right) \mathrm{d} s
\end{gathered}
$$

$$
\begin{aligned}
& \left.+\sum_{t_{k}<t} I_{k}\left(w_{0}\left(t_{k}\right)\right)\right] \mathrm{d} t \\
& +\int_{0}^{t} \varphi_{p}^{-1} \\
& \times\left(\int_{s}^{+\infty} q(\tau) f\left(\tau, w_{0}(\tau), w_{0}^{\prime}(\tau)\right) \mathrm{d} \tau+\varphi_{p}\left(x_{\infty}\right)\right) \mathrm{d} s \\
& +\sum_{t_{k}<t} I_{k}\left(w_{0}\left(t_{k}\right)\right) \\
& \leq \frac{1}{1-\int_{0}^{+\infty} g(t) \mathrm{d} t} \\
& \times \int_{0}^{+\infty} \operatorname{tg}(t) \mathrm{d} t \cdot \varphi_{p}^{-1} \\
& \times\left(\int_{0}^{+\infty} q(\tau) f\left(\tau, x(\tau), x^{\prime}(\tau)\right) \mathrm{d} \tau+\varphi_{p}\left(x_{\infty}\right)\right) \\
& +t \varphi_{p}^{-1}\left(\int_{0}^{+\infty} q(\tau) f\left(\tau, x(\tau), x^{\prime}(\tau)\right) \mathrm{d} \tau+\varphi_{p}\left(x_{\infty}\right)\right) \\
& +\frac{1}{1-\int_{0}^{+\infty} g(t) \mathrm{d} t}\left(a^{*}+b^{*} d\right) \\
& \leq 2^{1 /(p-1)}\left(\frac{1}{1-\int_{0}^{+\infty} g(t) \mathrm{d} t} \cdot \int_{0}^{+\infty} \operatorname{tg}(t) \mathrm{d} t\right) \\
& \times\left[\varphi_{p}^{-1}\left(\int_{0}^{+\infty} q(\tau) \mathrm{d} \tau\right) \varphi_{p}^{-1}\left(\varphi_{p}\left(\frac{d}{3 m}\right)\right)+x_{\infty}\right] \\
& +2^{1 /(p-1)} t\left[\varphi_{p}^{-1}\left(\int_{0}^{+\infty} q(\tau) \mathrm{d} \tau\right) \varphi_{p}^{-1}\left(\varphi_{p}\left(\frac{d}{3 m}\right)\right)+x_{\infty}\right] \\
& +\frac{1}{1-\int_{0}^{+\infty} g(t) \mathrm{d} t}\left(a^{*}+b^{*} d\right) \\
& \leq d+d t=w_{0}(t) \\
& w_{1}^{\prime}(t) \\
& =\left(A w_{0}\right)^{\prime}(t) \\
& =\varphi_{p}^{-1}\left(\int_{t}^{+\infty} q(s) f\left(s, w_{0}(s), w_{0}^{\prime}(s)\right) \mathrm{d} s+\varphi_{p}\left(x_{\infty}\right)\right) \\
& \leq 2^{1 /(p-1)}\left[\varphi_{p}^{-1}\left(\int_{0}^{+\infty} q(\tau) \mathrm{d} \tau\right) \varphi_{p}^{-1}\left(\varphi_{p}\left(\frac{d}{3 m}\right)\right)+x_{\infty}\right] \\
& \leq d \\
& =w_{0}^{\prime}(t), 0 \leq t<+\infty \text {. }
\end{aligned}
$$

So, by $(53)\left(A_{1}\right)-\left(A_{3}\right)$ we have

$$
\begin{gathered}
w_{2}(t)=\left(A w_{1}\right)(t) \leq\left(A w_{0}\right)(t)=w_{1}(t), \quad 0 \leq t<+\infty, \\
w_{2}^{\prime}(t)=\left(A w_{1}\right)^{\prime}(t) \leq\left(A w_{0}\right)^{\prime}(t)=\left(w_{1}\right)^{\prime}(t), \quad 0 \leq t<+\infty .
\end{gathered}
$$


By induction, we get that

$$
\begin{gathered}
w_{n+1}(t) \leq w_{n}(t), \\
w_{n+1}^{\prime}(t) \leq w_{n}^{\prime}(t), \\
0 \leq t<+\infty, \quad n=0,1,2, \ldots .
\end{gathered}
$$

Hence, we claim that $w_{n} \rightarrow w^{*}$ as $n \rightarrow \infty$. Applying the continuity of $A$ and $w_{n+1}=A w_{n}$, we get that $A w^{*}=w^{*}$.

Let $v_{0}(t)=0,0 \leq t<+\infty$, then $v_{0}(t) \in \bar{P}_{d}$. Let $v_{1}=$ $A v_{0}, v_{2}=A^{2} v_{0}$. By Lemma 6, we have that $v_{1} \in \bar{P}_{d}$ and $v_{2} \in$ $\bar{P}_{d}$. Denote

$$
v_{n+1}=A v_{n}=A^{n+1} v_{0}, \quad n=0,1,2, \ldots
$$

Since $A: \bar{P}_{d} \rightarrow \bar{P}_{d}$, we have that $v_{n} \in A\left(\bar{P}_{d}\right) \subset \bar{P}_{d}, n=$ $1,2,3, \ldots$ It follows from the complete continuity of $A$ that $\left\{v_{n}\right\}_{n=1}^{\infty}$ is a sequentially compact set. And, we assert that $\left\{v_{n}\right\}_{n=1}^{\infty}$ has a convergent subsequence $\left\{v_{n_{k}}\right\}_{k=1}^{\infty}$ and there exists $v^{*} \in \bar{P}_{d}$ such that $v_{n_{k}} \rightarrow v^{*}$.

Since $v_{1}=A v_{0} \in \bar{P}_{d}$, we have

$$
\begin{gathered}
v_{1}(t)=\left(A v_{0}\right)(t)=(A 0)(t) \geq 0, \quad 0 \leq t<+\infty, \\
v_{1}^{\prime}(t)=\left(A v_{0}\right)^{\prime}(t)=(A 0)^{\prime}(t) \geq 0=v_{0}^{\prime}(t), \quad 0 \leq t<+\infty .
\end{gathered}
$$

By $\left(A_{1}\right)-\left(A_{3}\right)$, we have

$$
\begin{array}{ll}
v_{2}(t)=\left(A v_{1}\right)(t) \geq(A 0)(t)=v_{1}(t), & 0 \leq t<+\infty, \\
v_{2}^{\prime}(t)=\left(A v_{1}\right)^{\prime}(t) \geq(A 0)^{\prime}(t)=v_{1}^{\prime}(t), & 0 \leq t<+\infty .
\end{array}
$$

By induction, we get that

$$
\begin{gathered}
v_{n+1}(t) \geq v_{n}(t), \\
v_{n+1}^{\prime}(t) \geq v_{n}^{\prime}(t), \\
0 \leq t<+\infty, \quad n=0,1,2, \ldots .
\end{gathered}
$$

Hence, we claim that $v_{n} \rightarrow v^{*}$ as $n \rightarrow \infty$. Applying the continuity of $A$ and $v_{n+1}=A v_{n}$, we get that $A v^{*}=v^{*}$.

Since $f(t, 0,0) \not \equiv 0,0 \leq t<\infty$, then the zero function is not the solution of BVP (4). Thus, $v^{*}$ is a positive solution of BVP (4). By Lemma 4 we know that $w^{*}$ and $v^{*}$ are positive, nondecreasing on $[0,+\infty)$ and concave solutions of the BVP (4).

We can easily get that Theorem 7 holds for $1<p<2$ in a similar manner.

Remark 8. The iterative schemes in Theorem 7 are $w_{0}(t)=d+$ $d t, w_{n+1}=A w_{n}=A^{n+1} w_{0}, n=0,1,2, \ldots$ and $v_{0}(t)=0, v_{n+1}=$ $A v_{n}=A^{n+1} v_{0}, n=0,1,2, \ldots$. They start off with a known simple linear function and the zero function respectively. This is convenient in application.

Theorem 9. Assume that $\left(H_{1}\right)-\left(H_{3}\right)$ hold, and there exist

$$
d_{n}>d_{n-1}>\cdots>d_{1}> \begin{cases}3 \Lambda, & \text { as } p \geq 2, \\ 3 \Lambda^{\prime}, & \text { as } 1<p<2\end{cases}
$$

such that
$\left(\mathrm{A}_{1}^{\prime}\right) f\left(t, x_{1}, y_{1}\right) \leq f\left(t, x_{2}, y_{2}\right)$ for any $0 \leq t<+\infty, 0 \leq$ $x_{1} \leq x_{2}, 0 \leq y_{1} \leq y_{2}$.

$\left(\mathrm{A}_{2}^{\prime}\right)$

$$
\begin{aligned}
& f(t,(1+t) u, v) \leq \begin{cases}\varphi_{p}\left(\frac{d_{k}}{3 m}\right), & \text { as } p \geq 2, \\
\varphi_{p}\left(\frac{d_{k}}{3 m^{\prime}}\right), & \text { as } 1<p<2\end{cases} \\
& (t, u, v) \in[0,+\infty) \times\left[0, d_{k}\right] \times\left[0, d_{k}\right], k=1,2, \ldots, n .
\end{aligned}
$$

$$
\left(\mathrm{A}_{3}^{\prime}\right) I_{k}\left(x_{1}\right) \leq I_{k}\left(x_{2}\right)(k=1,2, \ldots,) \text {, for any } 0 \leq x_{1} \leq x_{2} .
$$

Then, the boundary value problem (4) admits positive nondecreasing on $[0,+\infty)$ and concave solutions $w_{k}^{*}$ and $v_{k}^{*}$, such that $0<\left\|w_{k}^{*}\right\|_{D} \leq d_{k}$, and $\lim _{n \rightarrow \infty} w_{k n}=\lim _{n \rightarrow \infty} A^{n} w_{k 0}=w_{k}^{*}$, where

$$
w_{0}(t)=d_{k}+d_{k} t, \quad t \in J,
$$

and $0<\left\|v_{k}^{*}\right\|_{D} \leq d_{k}, \lim _{n \rightarrow \infty} v_{k n}=\lim _{n \rightarrow \infty} A^{n} v_{k 0}=v_{k}^{*}$, where $v_{0}(t)=0, t \in J$.

Remark 10. It is easy to see that $w^{*}$ and $v^{*}$ in Theorem 7 may coincide, and then the boundary value problem (4) has only one solution in $P$. Similarly, positive solutions $w_{k}^{*}$ and $v_{k}^{*}$ may also coincide.

\section{An Example}

Example 11. Consider the following impulsive integral boundary value problem:

$$
\begin{aligned}
& \left(\left|x^{\prime}\right| x^{\prime}\right)^{\prime}+e^{-6 t} f\left(t, x(t), x^{\prime}(t)\right)=0, \quad t \in J_{+}, \\
& \left.\Delta x\right|_{t=k}=\frac{1}{9}\left[\frac{1}{2^{k+2}} x(k)+\frac{1}{2^{k+1}}(1+x(k))^{1 / 6}\right], \\
& x(0)=\int_{0}^{+\infty} \frac{1}{(1+t)^{3}} x(t) \mathrm{d} t, \quad x^{\prime}(\infty)=\frac{\sqrt{2}}{3},
\end{aligned}
$$

where

$f(t, u, v)$

$$
= \begin{cases}\frac{1}{64}|\sin (101 t+20)|+\frac{1}{72}\left(\frac{u}{1+t}\right)^{3}+\frac{1}{10}\left(\frac{v}{20}\right), & u \leq 2, \\ \frac{1}{64}|\sin (101 t+20)|+\frac{1}{72}\left(\frac{2}{1+t}\right)^{3}+\frac{1}{10}\left(\frac{v}{20}\right), & u \geq 2 .\end{cases}
$$

It is clear that conditions $\left(\mathrm{H}_{1}\right),\left(\mathrm{A}_{1}\right)$, and $\left(\mathrm{A}_{3}\right)$ hold for $p=$ $3, q(t)=e^{-6 t}, g(t)=1 /(1+t)^{3}$. By direct computation, we obtain that

$$
\int_{0}^{+\infty} q(t) \mathrm{d} t=\frac{1}{6}, \quad \int_{0}^{+\infty} \varphi_{p}^{-1}\left(\int_{s}^{+\infty} q(\tau) \mathrm{d} \tau\right) \mathrm{d} s=\frac{\sqrt{3}}{18},
$$

which implies that $\left(\mathrm{H}_{2}\right)$ holds. 
Obviously, $I_{k} \in C(J, J)$. Using a simple inequality

$$
(1+u)^{\alpha} \leq 1+\alpha u, \quad \forall u \geq 0,0<\alpha<1,
$$

we get that

$$
\begin{aligned}
I_{k}(x(k)) & \leq \frac{1}{9}\left[\frac{1}{2^{k+2}} x(k)+\frac{1}{2^{k+1}}\left(1+\frac{1}{6} x(k)\right)\right] \\
& \leq \frac{1}{9} \cdot \frac{1}{2^{k+1}}+\frac{2}{27} \cdot \frac{1}{2^{k+1}} x(k) .
\end{aligned}
$$

Thus, $\left(\mathrm{H}_{3}\right)$ holds for $a_{k}=(1 / 9) \cdot\left(1 / 2^{k+1}\right), b_{k}=(2 / 27) \cdot\left(1 / 2^{k+1}\right)$. Considering that

$$
\begin{gathered}
\int_{0}^{+\infty} g(t) \mathrm{d} t=\int_{0}^{+\infty} \frac{1}{(1+t)^{3}} \mathrm{~d} t=\frac{1}{2}, \\
\int_{0}^{+\infty} \operatorname{tg}(t) \mathrm{d} t=\int_{0}^{+\infty} \frac{t}{(1+t)^{3}} \mathrm{~d} t=\frac{1}{2}, \\
\varphi_{p}^{-1}\left(\int_{0}^{+\infty} q(\tau) \mathrm{d} \tau\right)=\varphi_{p}^{-1}\left(\frac{1}{6}\right)=\frac{\sqrt{6}}{6},
\end{gathered}
$$

we can obtain that

$$
\begin{gathered}
a^{*}=\sum_{k=1}^{\infty} a_{k}=\frac{1}{18}, \\
b^{*}=\frac{2}{27} \sum_{k=1}^{\infty} \frac{1+k}{2^{k+1}}=\frac{2}{27}\left(\sum_{k=1}^{\infty} \frac{1}{2^{k+1}}+\sum_{k=1}^{\infty} \frac{k}{2^{k+1}}\right)=\frac{1}{9}, \\
m=\sqrt{3}, \quad n=2, \quad \Lambda=2 .
\end{gathered}
$$

Take $d=8$. In this case, we have

$$
\varphi_{p}\left(\frac{d}{3 m}\right)=\varphi_{p}\left(\frac{8}{3 \sqrt{3}}\right)=\frac{64}{27} .
$$

On the other hand, nonlinear term $f$ satisfies

$$
\begin{aligned}
& f(t,(1+t) u, v) \\
& \leq \frac{1}{64}+\frac{1}{9}+\frac{1}{25}=\frac{2401}{14400}, \quad t \in[0,+\infty), u, v \in[0,8]
\end{aligned}
$$

which means that $\left(A_{2}\right)$ holds. Thus, we have checked that all the conditions of Theorem 7 are satisfied. Therefore, the conclusion of Theorem 7 holds.

\section{Acknowledgments}

This paper is supported financially by the Foundation for Outstanding Middle-Aged and Young Scientists of Shandong Province (BS2010SF004), the Project of Shandong Province Higher Educational Science and Technology Program (no. J10LA53), and the National Natural Science Foundation of China (10971179).

\section{References}

[1] V. Lakshmikantham, D. D. Bainov, and P. S. Simeonov, Theory of Impulsive Differential Equations, vol. 6 of Series in Modern Applied Mathematics, World Scientific Publishing, Teaneck, NJ, USA, 1989.

[2] D. D. Bainov and P. S. Simeonov, Systems with Impulse Effect: Stability, Theory and Application, Ellis Horwood Series: Mathematics and its Applications, Ellis Horwood, Chichester, UK, 1989.

[3] A. M. Samoĭlenko and N. A. Perestyuk, Impulsive Differential Equations, vol. 14 of World Scientific Series on Nonlinear Science. Series A: Monographs and Treatises, World Scientific Publishing, River Edge, NJ, USA, 1995.

[4] R. P. Agarwal and D. O'Regan, Infinite Interval Problems for Differential, Difference and Integral Equations, Kluwer Academic Publishers, Dordrecht, The Netherland, 2001.

[5] S. Z. Chen and Y. Zhang, "Singular boundary value problems on a half-line," Journal of Mathematical Analysis and Applications, vol. 195, no. 2, pp. 449-468, 1995.

[6] X. Liu, "Solutions of impulsive boundary value problems on the half-line," Journal of Mathematical Analysis and Applications, vol. 222, no. 2, pp. 411-430, 1998.

[7] M. Zima, "On positive solutions of boundary value problems on the half-line," Journal of Mathematical Analysis and Applications, vol. 259, no. 1, pp. 127-136, 2001.

[8] J. Li and J. J. Nieto, "Existence of positive solutions for multipoint boundary value problem on the half-line with impulses," Boundary Value Problems, vol. 2009, Article ID 834158, 12 pages, 2009.

[9] G. L. Karakostas and P. Ch. Tsamatos, "Multiple positive solutions of some Fredholm integral equations arisen from nonlocal boundary-value problems," Electronic Journal of Differential Equations, vol. 30, pp. 1-17, 2002.

[10] G. L. Karakostas and P. Ch. Tsamatos, "Existence of multiple positive solutions for a nonlocal boundary value problem," Topological Methods in Nonlinear Analysis, vol. 19, no. 1, pp. 109121, 2002.

[11] J. R. L. Webb and G. Infante, "Positive solutions of nonlocal boundary value problems: a unified approach," Journal of the London Mathematical Society, vol. 74, no. 3, pp. 673-693, 2006.

[12] J. R. L. Webb and G. Infante, "Positive solutions of nonlocal boundary value problems involving integral conditions," Nonlinear Differential Equations and Applications, vol. 15, no. 1-2, pp. 45-67, 2008.

[13] Z. Yang, "Existence and nonexistence results for positive solutions of an integral boundary value problem," Nonlinear Analysis. Theory, Methods \& Applications A, vol. 65, no. 8, pp. 1489-1511, 2006.

[14] Z. Yang, "Positive solutions of a second-order integral boundary value problem," Journal of Mathematical Analysis and Applications, vol. 321, no. 2, pp. 751-765, 2006.

[15] M. Feng, "Existence of symmetric positive solutions for a boundary value problem with integral boundary conditions," Applied Mathematics Letters, vol. 24, no. 8, pp. 1419-1427, 2011.

[16] M. Feng, X. Zhang, and W. Ge, "New existence results for higher-order nonlinear fractional differential equation with integral boundary conditions," Boundary Value Problems, vol. 2011, Article ID 720702, 20 pages, 2011.

[17] B. Ahmad, A. Alsaedi, and B. S. Alghamdi, "Analytic approximation of solutions of the forced Duffing equation with 
integral boundary conditions," Nonlinear Analysis. Real World Applications, vol. 9, no. 4, pp. 1727-1740, 2008.

[18] M. Feng, X. Liu, and H. Feng, “The existence of positive solution to a nonlinear fractional differential equation with integral boundary conditions," Advances in Difference Equations, vol. 2011, Article ID 546038, 14 pages, 2011.

[19] P. Kang, Z. Wei, and J. Xu, "Positive solutions to fourthorder singular boundary value problems with integral boundary conditions in abstract spaces," Applied Mathematics and Computation, vol. 206, no. 1, pp. 245-256, 2008.

[20] X. Zhang and W. Ge, "Symmetric positive solutions of boundary value problems with integral boundary conditions," Applied Mathematics and Computation, vol. 219, no. 8, pp. 3553-3564, 2012.

[21] X. Zhang, X. Yang, and M. Feng, "Minimal nonnegative solution of nonlinear impulsive differential equations on infinite interval," Boundary Value Problems, vol. 2011, Article ID 684542, 15 pages, 2011.

[22] D. Guo, "Existence of positive solutions for $n$ th-order nonlinear impulsive singular integro-differential equations in Banach spaces," Nonlinear Analysis. Theory, Methods \& Applications A, vol. 68, no. 9, pp. 2727-2740, 2008.

[23] Y. Liu, "Existence and unboundedness of positive solutions for singular boundary value problems on half-line," Applied Mathematics and Computation, vol. 144, no. 2-3, pp. 543-556, 2003.

[24] Y. Liu, "Boundary value problems for second order differential equations on unbounded domains in a Banach space," Applied Mathematics and Computation, vol. 135, no. 2-3, pp. 569-583, 2003.

[25] B. Yan, D. O'Regan, and R. P. Agarwal, "Unbounded solutions for singular boundary value problems on the semi-infinite interval: upper and lower solutions and multiplicity," Journal of Computational and Applied Mathematics, vol. 197, no. 2, pp. 365386, 2006.

[26] B. Yan and Y. Liu, "Unbounded solutions of the singular boundary value problems for second order differential equations on the half-line," Applied Mathematics and Computation, vol. 147, no. 3, pp. 629-644, 2004.

[27] H. Lian, H. Pang, and W. Ge, "Triple positive solutions for boundary value problems on infinite intervals," Nonlinear Analysis. Theory, Methods \& Applications A, vol. 67, no. 7, pp. 21992207, 2007.

[28] S. Liang and J. Zhang, "The existence of countably many positive solutions for some nonlinear three-point boundary problems on the half-line," Nonlinear Analysis. Theory, Methods \& Applications A, vol. 70, no. 9, pp. 3127-3139, 2009.

[29] L. Liu, Z. Liu, and Y. Wu, "Infinite boundary value problems for $n$ th-order nonlinear impulsive integro-differential equations in Banach spaces," Nonlinear Analysis. Theory, Methods \& Applications A, vol. 67, no. 9, pp. 2670-2679, 2007.

[30] D.-X. Ma, Z.-J. Du, and W.-G. Ge, "Existence and iteration of monotone positive solutions for multipoint boundary value problem with $p$-Laplacian operator," Computers \& Mathematics with Applications, vol. 50, no. 5-6, pp. 729-739, 2005.

[31] B. Sun, J. Zhao, P. Yang, and W. Ge, "Successive iteration and positive solutions for a third-order multipoint generalized right-focal boundary value problem with $p$-Laplacian," Nonlinear Analysis. Theory, Methods \& Applications A, vol. 70, no. 1, pp. 220-230, 2009.

[32] B. Sun, A. Yang, and W. Ge, "Successive iteration and positive solutions for some second-order three-point $p$-Laplacian boundary value problems," Mathematical and Computer Modelling, vol. 50, no. 3-4, pp. 344-350, 2009.

[33] Y. S. Liu, "A boundary value problem for a second-order differential equation on an unbounded domain," Acta Analysis Functionalis Applicata, vol. 4, no. 3, pp. 211-216, 2002 (Chinese).

[34] C. Corduneanu, Integral Equations and Stability of Feedback Systems, Academic Press, New York, NY, USA, 1973. 


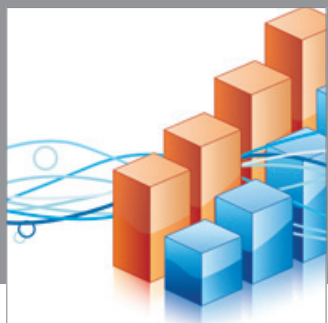

Advances in

Operations Research

mansans

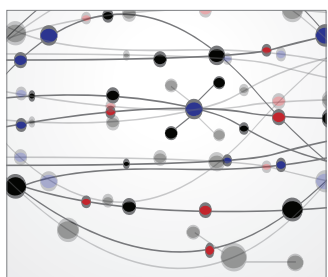

The Scientific World Journal
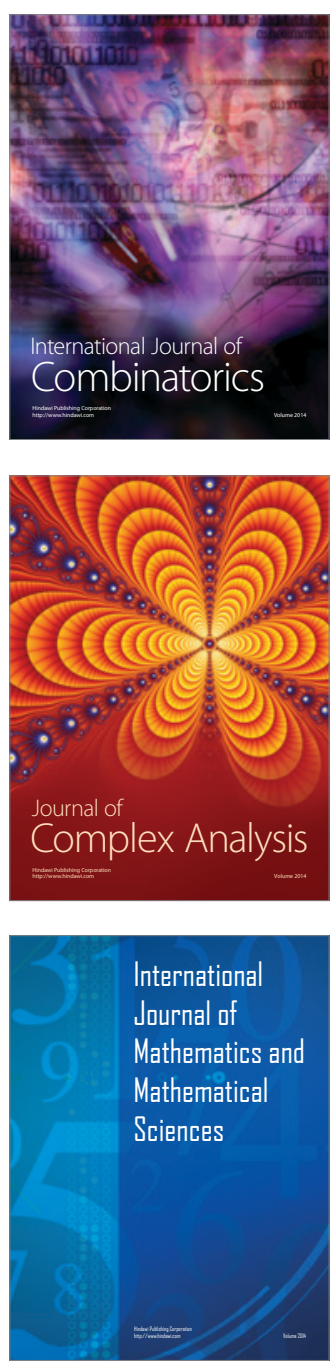
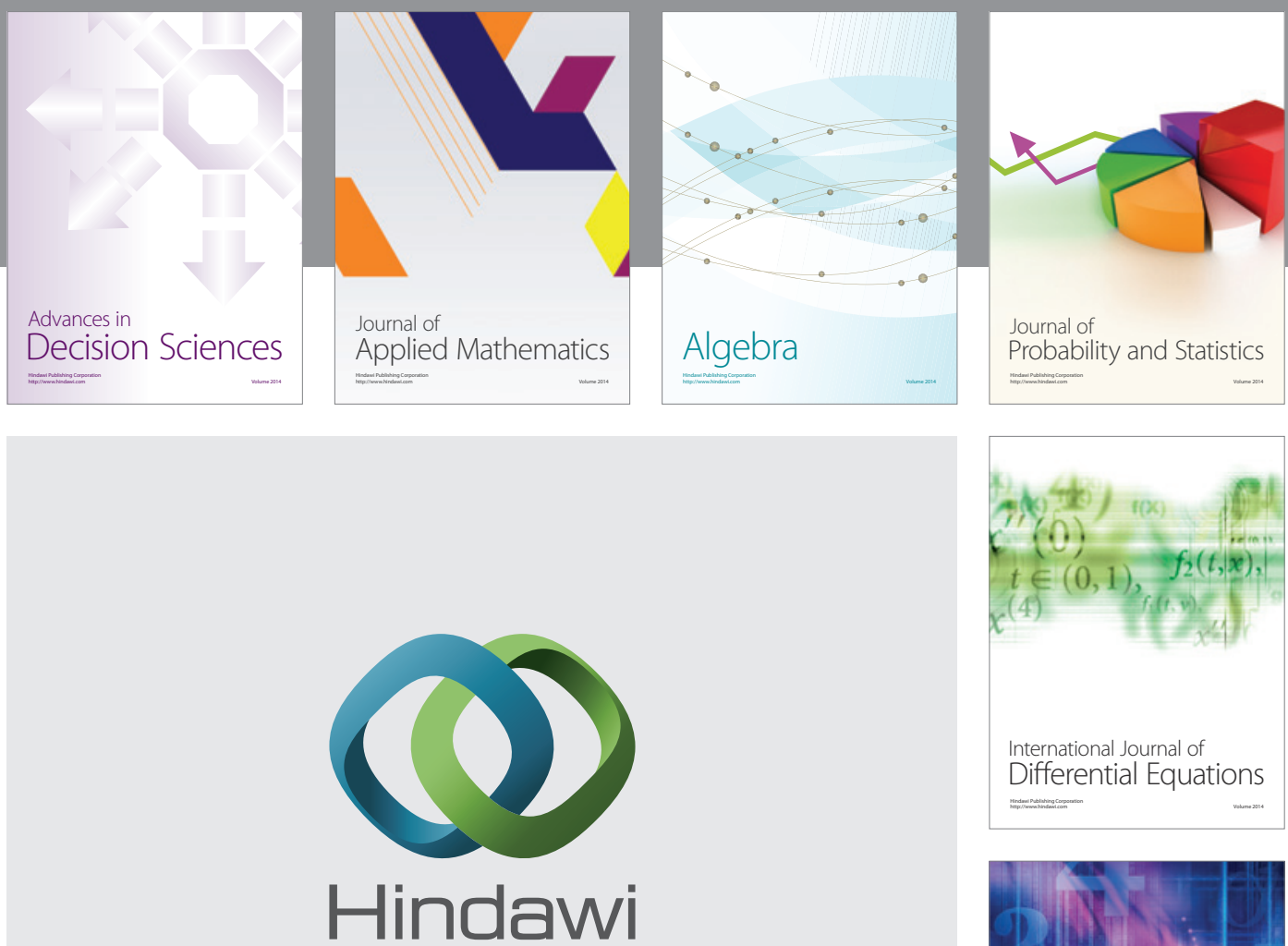

Submit your manuscripts at http://www.hindawi.com
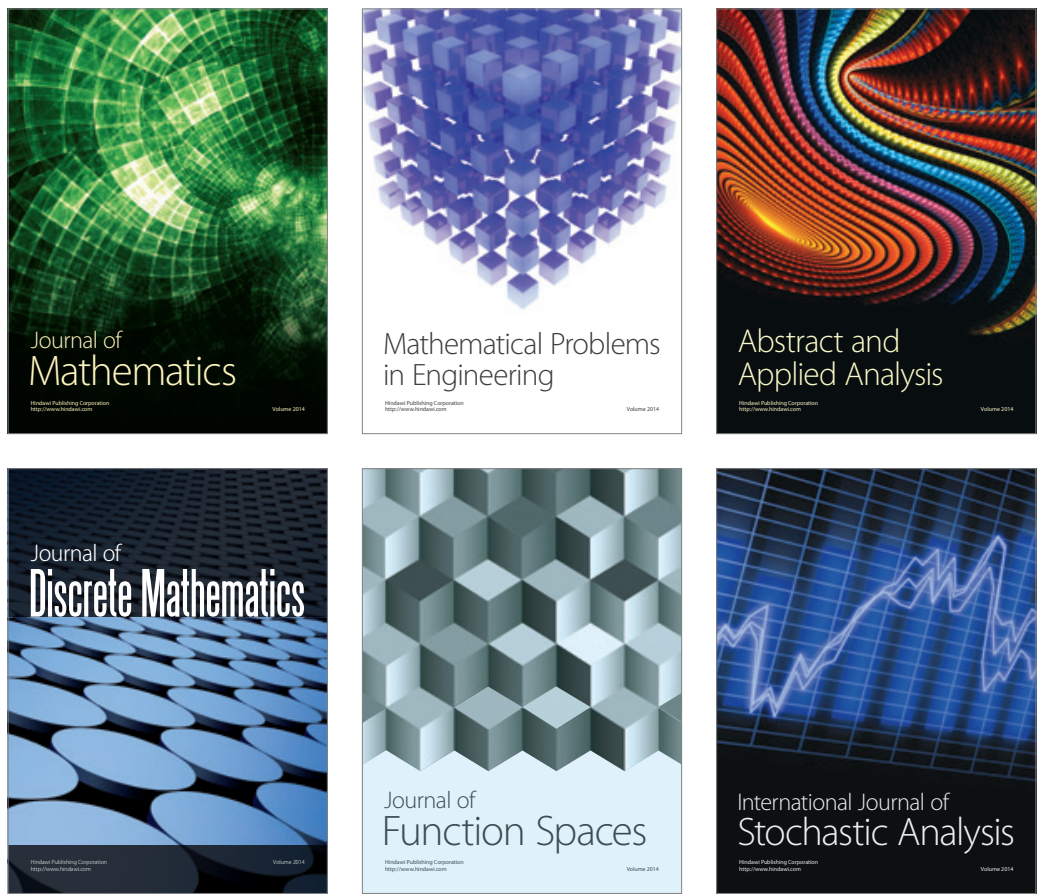

Journal of

Function Spaces

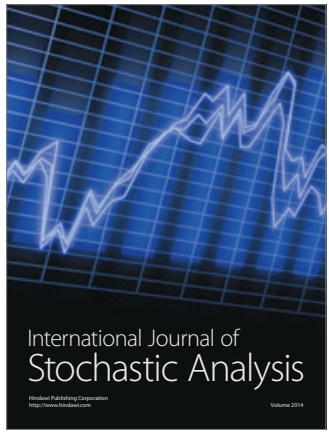

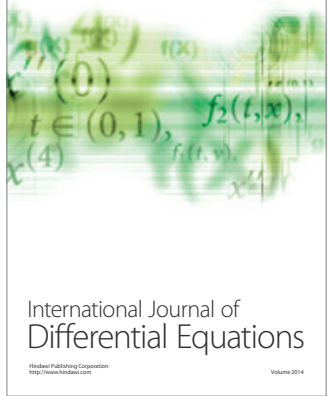
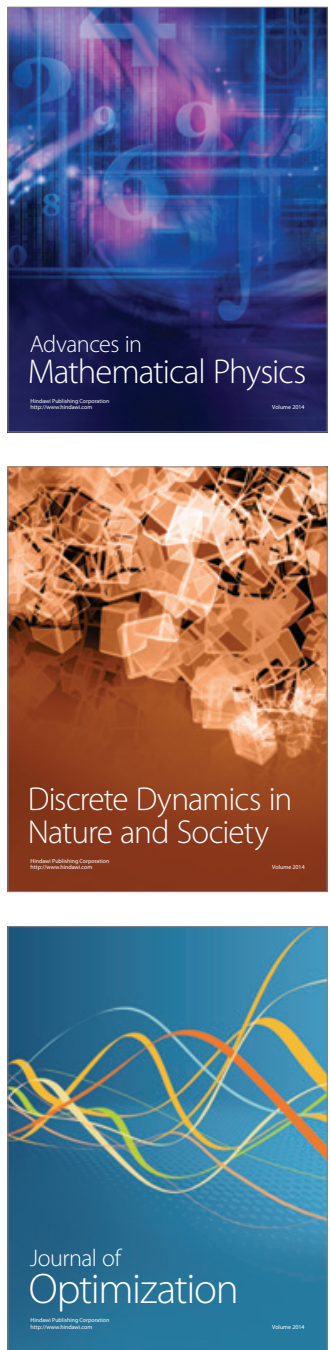